\title{
Grouping, Overlap, and Generalized Bientropic Functions for Fuzzy Modeling of Pairwise Comparisons
}

\author{
Humberto Bustince, Member, IEEE, Miguel Pagola, Radko Mesiar, Eyke Hüllermeier, \\ and Francisco Herrera, Member, IEEE
}

\begin{abstract}
In this paper, we propose new aggregation functions for the pairwise comparison of alternatives in fuzzy preference modeling. More specifically, we introduce the concept of a grouping function, i.e., a specific type of aggregation function that combines two degrees of support (weak preference) into a degree of information or, say, a degree of comparability between two alternatives, and we relate this new concept to that of incomparability. Grouping functions of this type complement the existing concept of overlap functions in a natural way, since the latter can be used to turn two degrees of weak preference into a degree of indifference. We also define the so-called generalized bientropic functions that allow for a unified representation of overlap and grouping functions. Apart from analyzing mathematical properties of these types of functions and exploring relationships between them, we elaborate on their use in fuzzy preference modeling and decision making. We present an algorithm to elaborate on an alternative preference ranking that penalizes those alternatives for which the expert is not sure of his/her preference.
\end{abstract}

Index Terms-Decision making, generalized bientropic function, grouping function, incomparability, overlap function, pairwise comparison, preference relations.

\section{INTRODUCTION}

$\mathbf{F}$ ORMAL models for dealing with preferences have a long tradition in diverse research disciplines, ranging from economics and philosophy to operations research and statistics [24]. The two approaches prevailing the literature on choice and decision theory are based, respectively, on the two perhaps most

Manuscript received September 23, 2010; revised May 3, 2011 and July 30, 2011; accepted September 28, 2011. Date of publication October 25, 2011; date of current version May 27, 2012. The work of H. Bustince and M. Pagola was supported by the Spanish Ministry of Science under Grant TIN2010-15055. The work of R. Mesiar was supported by Grant APVV-0073-10 and Grant P GACR P 402/11/0378. The work of F. Herrera was supported by the Spanish Ministry of Science under Grant TIN2011-28488.

H. Bustince and M. Pagola are with the Department of Automatic and Computation, Public University of Navarra, Pamplona, Navarra 31006, Spain (e-mail bustince@unavarra.es; miguel.pagola@unavarra.es).

R. Mesiar is with the Department of Mathematics and Descriptive Geometry, Slovak University of Technology, Vazovova, Bratislava 81243, Slovakia, and also with the Institute of Theory of Information and Automation, Czech Academy of Sciences, Prague 11720, Czech Republic (e-mail: mesiar@math.sk).

E. Hüllermeier is with the Department of Mathematics and Computer Science, Philipps-Universität Marburg, Marburg D-35032, Germany (e-mail: eyke@mathematik.uni-marburg.de).

F. Herrera is with the Department of Computation and Artificial Intelligence, Universidad de Granada, Granada 18010, Spain (e-mail: herrera@decsai.ugr.es).

Digital Object Identifier 10.1109/TFUZZ.2011.2173581 natural ways for expressing preferences, namely by evaluating individual alternatives and by comparing (pairs of) competing alternatives. While the former approach leads to preference models in the form of (numerical) utility functions, the latter employs (binary) relations in order to express (comparative) preferences in a qualitative way.

The relational approach to preference modeling is arguably more convenient from a knowledge acquisition point of view, especially since people often find it easier to compare two alternatives than to assess single alternatives in terms of numerical utility degrees. In fact, a number of well-known methods for decision making are based on pairwise comparisons of this type, including the Saatys analytical hierarchy process [31] and decision making based on fuzzy preference relations [1], [9], [11], [12], [16], [29], [35].

In fuzzy preference modeling, an indifference relation is usually derived from a weak preference relation $\mathcal{R}$ by means of a t-norm combination, i.e., $\mathcal{I}(A, B)=T(\mathcal{R}(A, B), \mathcal{R}(B, A))$, and it is a measure of the simultaneous fulfillment of $\mathcal{R}(A, B)$ and $\mathcal{R}(B, A)$. Likewise, incomparability is modeled in terms of a logical expression of the form, i.e., $\mathcal{J}(A, B)=$ $T(N(\mathcal{R}(A, B)), N(\mathcal{R}(B, A)))$, meaning that $A$ and $B$ cannot be compared if neither $A$ is (weakly) preferred to $B$ nor if it is the other way around ( $N$ is a negation operator) [17], [30].

However, despite the usefulness and soundness of this approach, one may argue that, at least in the context of the pairwise comparison of alternatives, the use of the standard fuzzy logical operators to combine weak preference or, say, support degrees is not compulsory. For example, there is, in principle, no strong reason to require associativity of the combination function, since by definition, a pairwise comparison refers to only two alternatives (and the result of the comparison is not an alternative).

Taking into account that Fodor and Roubens' axiomatization of the preference structures only demands independence, monotonicity, and symmetry and that associativity is not required, in this study, we aim to present new construction methods for the concepts of indifference and incomparability that do not forcibly depend on the use of t-norms.

To this end, we continue the work that was started in [6], where the concept of an overlap function was introduced. Consider the problem of assigning an object $q$ to either class $A$ (which corresponds to the event $q \in A$ ) or class $B$ (corresponding to $q \in B$ ). Roughly speaking, a degree of overlap between two support degrees $\mathcal{R}(A, B)$ and $\mathcal{R}(B, A)$ is the amount of 
simultaneous evidence in favor of $q \in A$ and $q \in B$. With regard to the assignment of $q$ to one of the classes $A$ or $B$, it may hence serve as a measure of indifference in the sense of [17]. Here, we complement this function by what we call a grouping function. The latter is supposed to measure the amount of evidence in favor of either of the two classes $A$ and $B$. Thus, its negation may serve as a measure of incomparability. As a unifying element, we introduce the concept of a generalized bientropic function for the representation of the uncertainty that is involved in assigning $q$ to class $A$ or $B$ and show that both the overlap and the grouping function can be derived from a generalized bientropic function.

These considerations have led us to pose the following objectives.

1) Define the concept of grouping function.

2) Define the concept of generalized bientropic function.

3) Analyze the connections of these concepts with overlap functions.

4) Define the concepts of indifference and incomparability in the sense of [17] and [30] and in terms of overlap functions, grouping functions, and bientropic functions.

A problem arises in the pairwise comparison of alternatives in fuzzy preference modeling when the expert provides a value for the preference of alternative $A$ over alternative $B$ close to 0.5 , i.e., when the expert is not sure of which alternative he or she prefers. For this situation, we propose an algorithm that makes use of the concept of a generalized bientropic function, since these functions allow us to penalize values around 0.5 (just by taking the minimal entropy). In the algorithm that we propose, we are also going to penalize indifference of the experts in the preference of alternatives, as well as the incomparability between them.

This paper is organized as follows. In Section II, we introduce the basic concepts that we are going to use later. In Section III, we define grouping functions, and we study their properties. In Section IV, we introduce the concept of generalized bientropic functions and study their main properties. In Section V, we consider connections between generalized bientropic functions, overlap functions, and grouping functions. In Section VI, we elaborate on differences and similarities between the concepts of indifference, incomparability, overlap, grouping, and generalized bientropic function. We present an algorithm for the exploitation phase in decision-making problems that allows us to select the best alternative by minimization of an expression in terms of generalized bientropic functions, indifference, and incomparability. In Section VII, we present some open problems. Finally, in Section VIII, we present some conclusions.

\section{PREliminary Definitions}

We start by recalling some basic concepts that we are going to use in our subsequent developments.

\section{A. Negations, Automorphisms, and Aggregation Functions}

A strict negation (see [34]) is a continuous and strictly decreasing mapping, i.e., $N:[0,1] \rightarrow[0,1]$, such that $N(0)=1$ and $N(1)=0$. A strong negation is an involutive strict nega- tion, i.e., a strict negation $N$, such that $N(N(x))=x$ for all $x \in[0,1]$.

Given a strong negation $N$, an equilibrium point $e \in(0,1)$ of that negation is characterized by the property, i.e., $N(e)=e$. It is easy to show that a strong negation has a unique equilibrium point.

An automorphism (see [3] and [4]) of the unit interval is a bijective mapping, i.e., $\varphi:[0,1] \rightarrow[0,1]$, which is strictly increasing $(\varphi(0)=0$ and $\varphi(1)=1)$. Observe that, in particular, any automorphism is continuous.

For us, an aggregation function (see [2] and [18]) will be a binary increasing mapping $M:[0,1]^{2} \rightarrow[0,1]$, such that $M(0,0)=0$ and $M(1,1)=1$. The well-known instances of aggregation functions are t-norms (see [25]). An aggregation function is conjunctive if it is bounded by the minimum, i.e., if $M\left(x_{1}, x_{2}\right) \leq \min \left(x_{1}, x_{2}\right)$. We say that an aggregation function does not have divisors of zero if $M\left(x_{1}, x_{2}\right)=0$, which implies that $x_{1}=0$ or that $x_{2}=0$.

\section{B. Fuzzy Relations}

Definition 1 [17]: Let $X=\left\{x_{1}, \ldots, x_{n}\right\}$ be a referential set. A binary fuzzy relation $R$ on $X$ is defined as a fuzzy subset of $X \times X$, i.e., $R: X \times X \rightarrow[0,1]$. The value, i.e., $R\left(x_{i}, x_{j}\right)=$ $R_{i j}$, denotes the degree of membership of the element $\left(x_{i}, x_{j}\right) \in$ $X \times X$ in $R$ (see [10] and [17]).

We will say that the fuzzy relation $R$ satisfies the property of reciprocity (or that $R$ is reciprocal) if $R_{i j}+R_{j i}=1$ for all $i, j \in\{1, \ldots, n\}$. For this kind of relations, it is common not to define the main diagonal elements (see [19] and [23]). We will denote by $\mathcal{F} R(X \times X)$ the set of all binary fuzzy relations on $X$.

\section{Overlap Functions}

Recall the situation that is outlined in Section I, in which an object $q$ must be assigned to class $A$ or class $B$. Moreover, suppose that we are given corresponding degrees of support (weak preference), $x=\mu_{A}(q)$ and $y=\mu_{B}(q)$, respectively, and we are interested in the degree $z$ to which $A$ and $B$ are supported simultaneously. More specifically, we are interested in a function, i.e., $F:[0,1]^{2} \rightarrow[0,1]$, such that $z=F(x, y)$. A natural candidate for $F$ is an overlap function as introduced in [6]. An overlap function is essentially characterized by symmetry and natural boundary and monotonicity properties.

Definition 2 [6]: An overlap function is a mapping, i.e., $G_{O}:[0,1]^{2} \rightarrow[0,1]$, such that

$\left.G_{O} 1\right) G_{O}(x, y)=G_{O}(y, x)$ for all $x, y \in[0,1]$;

$\left.G_{O} 2\right) G_{O}(x, y)=0$ if and only if $x=0$ or $y=0$;

$\left.G_{O} 3\right) G_{O}(x, y)=1$ if and only if $x=y=1$;

$\left.G_{O} 4\right) G_{O}$ is nondecreasing;

$\left.G_{O} 5\right) G_{O}$ is continuous.

Example 1: For any $p>0$, the mapping, i.e., $G_{O}(x, y)=$ $(\min (x, y))^{p}$, is an overlap function. Note that, for $p \neq 1$, this overlap function is not associative. 
A deep study of overlap functions has been developed in [6]. In particular, the following theorem and some construction methods were considered, as well as the relation between the overlap function and the analytic properties, such as migrativity or homogeneity.

Theorem 1 [6]: If a t-norm $T$ is an overlap function, then $T$ belongs to one of the following three types.

1) $T=T_{M}$.

2) $T$ is strict.

3) $T$ is the ordinal sum of the family $\left\{\left(\left[a_{m}, b_{m}\right], T_{m}\right)\right\}$, with all the $T_{m}$ being continuous Archimedean and such that if $a_{m_{0}}=0$ for some $m_{0}$, then $T_{m_{0}}$ is necessarily a strict t-norm.

Note that overlap functions form a convex class, whereas t-norms do not. Moreover, even the convex closure of the class of continuous t-norms with no zero divisors (which are the same as associative overlap functions) is a proper subclass of all overlap functions. Another important fact is that overlap functions are preserved under outer and inner transformations by means of automorphisms, i.e., if $\varphi_{1}, \varphi_{2}:[0,1] \rightarrow[0,1]$ are automorphisms, and $G_{O}:[0,1]^{2} \rightarrow[0,1]$ is an overlap function, then

$$
\begin{aligned}
& H_{O}:[0,1]^{2} \rightarrow[0,1] \text { given by } \\
& H_{O}(x, y)=\varphi_{1}\left(G_{O}\left(\varphi_{2}(x), \varphi_{2}(y)\right)\right)
\end{aligned}
$$

is also an overlap function.

The possible nonassociativity of overlap functions reflects the fact that they can combine the knowledge of several experts aggregating their individual overlap functions, e.g., as a convex sum (weighted arithmetic mean), or their log-convex sum (weighted geometric mean). The extension of Definition 2 from the binary case to $n$-dimensional overlap functions is straightforward, modifying $\left(G_{O} 1\right)$ into the symmetry of $n$-ary functions, $\left(G_{O} 2\right)$, requiring that $G_{O}\left(x_{1}, \ldots, x_{n}\right)=0$ if and only if $x_{i}=0$ for some $i \in\{1, \ldots, n\}$, and modifying $\left(G_{O} 3\right)$ into $G_{O}\left(x_{1}, \ldots, x_{n}\right)=1$ if and only if $x_{1}=\cdots=x_{n}=1$. Again, continuous t-norms (their $n$-ary forms) and their convex combinations are prototypical examples of $n$-ary overlap functions. Note that in our contribution we will work with binary overlap (and related) functions only.

\section{GROUPING FunCTIONS}

In this section, we introduce the concept of a grouping function as a natural complement of an overlap function. Given two degrees of support, i.e., $x=\mu_{A}(q)$ and $y=\mu_{B}(q)$, a grouping function is supposed to yield a degree $z$ to which the combination (grouping) of the two classes $A$ and $B$ is supported, i.e., either $A$ or $B$ or both.

Definition 3: A mapping $G_{G}:[0,1]^{2} \rightarrow[0,1]$ is a grouping function if it satisfies the following conditions.

$\left.G_{G} 1\right) G_{G}(x, y)=G_{G}(y, x)$ for all $x, y \in[0,1]$.

$\left.G_{G} 2\right) \quad G_{G}(x, y)=0$ if and only if $x=y=0$.

$\left.G_{G} 3\right) G_{G}(x, y)=1$ if and only if $x=1$ or $y=1$.

$\left.G_{G} 4\right) G_{G}$ is nondecreasing.

$\left.G_{G} 5\right) G_{G}$ is continuous.
Remark: Observe that a grouping function is a particular type of binary aggregation function. Moreover, as in the case of overlap functions, the extension of Definition 3 from the binary case to $n$-dimensional grouping functions is straightforward, modifying $\left(G_{G} 1\right)$ into the symmetry of $n$-ary functions, $\left(G_{G} 2\right)$ requiring that $G_{G}\left(x_{1}, \ldots, x_{n}\right)=0$ if and only if $x_{1}=\cdots=x_{n}=0$ and modifying $\left(G_{G} 3\right)$ into $G_{G}\left(x_{1}, \ldots, x_{n}\right)=1$ if and only if $x_{i}=1$ for some $i \in\{1, . ., n\}$. Again, continuous t-conorms (their $n$-ary forms) and their convex combinations are prototypical examples of $n$-ary grouping functions.

Example 2: The following binary aggregation functions are examples of grouping functions:

$$
\begin{aligned}
G_{G}(x, y) & =(\max (x, y))^{k} \text { with } k>0 \\
G_{G}(x, y) & =\frac{\max (x, y)}{\max (x, y)+\sqrt{(1-x)(1-y)}} .
\end{aligned}
$$

In the following sections, we establish some connections between grouping functions and overlap functions.

The following theorem shows the relation between grouping functions and overlap functions.

Theorem 2: Let $G_{O}$ be an overlap function, and let $N$ be a strict negation. Then

$$
G_{G}(x, y)=N\left(G_{O}(N(x), N(y))\right)
$$

is a grouping function. Reciprocally, if $G_{G}$ is a grouping function, then

$$
G_{O}(x, y)=N\left(G_{G}(N(x), N(y))\right)
$$

is an overlap function.

Proof: $\quad\left(G_{G} 1\right)$ The proof is direct. $\left(G_{G} 2\right), \quad G_{G}(x, y)=0=N\left(G_{O}(N(x)\right.$, $N(y))$ ) if and only if $G_{O}(N(x), N(y))=1$ if and only if $N(x)=N(y)=1$ if and only if $x=y=0 .\left(G_{G} 3\right)$, $G_{G}(x, y)=1=N\left(G_{O}(N(x), N(y))\right) \quad$ if and only if $G_{O}(N(x), N(y))=0$ if and only if $N(x)=0$ or $N(y)=0$ if and only if $x=1$ or $y=1 .\left(G_{G} 4\right)$ and $\left(G_{G} 5\right)$ are direct.

Corollary 1: Let

$$
G_{G}^{\text {Sup }}(x, y)=\left\{\begin{array}{l}
0 \text { if } x=y=0 \\
1 \text { in other case }
\end{array}\right.
$$

and

$$
G_{G}^{\operatorname{Inf}}(x, y)=\left\{\begin{array}{l}
1 \text { if } \max (x, y)=1 \\
0 \text { in other case. }
\end{array}\right.
$$

Then, the pointwise supremum of all grouping functions is given by $G_{G}^{\text {Sup }}$ and the pointwise infimum is given by $G_{G}^{\operatorname{Inf}}$. In particular

$$
G_{G}^{\operatorname{Inf}}(x, y) \leq G_{G}(x, y) \leq G_{G}^{\mathrm{Sup}}(x, y) \text { for all } x, y \in[0,1] .
$$

Proof: The proof is direct.

Comment 1: Let $G_{O}$ be an overlap function and $G_{G}$ be a grouping function. Then, it never happens that $G_{G}(x, y) \leq$ $G_{O}(x, y)$ for all $x, y \in[0,1]$. Just observe that, evidently, for any $G_{G}$ and $G_{O}$, it holds that $G_{G}(1,0)=1>0=G_{O}(1,0)$, excluding the possibility that $G_{G} \leq G_{O}$. 
Theorem 3: Let $G_{G}$ be an associative grouping function. Then, $G_{G}$ is a t-conorm.

Proof: We only need to see that 0 is the neutral element of $G_{G}$. From the continuity of $G_{G}$ and the fact that $G_{G}(1,0)=1$, $G_{G}(0,0)=0$, it follows that, for any $\left.x \in\right] 0,1[$, there exists $y \in] 0,1\left[\right.$, such that $x=G_{G}(y, 0)$. However, then, $G_{G}(x, 0)=$ $G_{G}\left(G_{G}(y, 0), 0\right)=G_{G}\left(y, G_{G}(0,0)\right)=G_{G}(y, 0)=x$, and similarly, $G_{G}(0, x)=x$.

To see that the converse of Theorem 3 does not hold, it is enough to consider discontinuous t-conorms.

Note that, like in the case of overlap functions, grouping functions form a convex class, which is closed under outer and inner automorphism transformations. Moreover, there exist functions other than t-conorms that are grouping functions; see (1) and (2).

\section{GENERALIZED BIENTROPIC FUNCTIONS}

Since overlap and grouping both measure specific types of uncertainty with regard to the class assignment of an object $q$, one may wonder whether these concepts can be unified in one way or the other. As will be shown later on, this is indeed possible, thanks to the concept of generalized bientropic functions as introduced in this section. The definition of this type of function builds on the normal $E_{N}$-functions that are introduced in [7].

Definition 4 [7]: A function $E_{N}:[0,1] \rightarrow[0,1]$ is called a normal $E_{N}$-function that is associated with the strong negation $N$ if it satisfies the following conditions.

1) $E_{N}(x)=0$ if and only if $x=0$ or $x=1$.

2) $E_{N}(x)=1$ if and only if $x=e$, where $e$ is the equilibrium point of $N$.

3) $E_{N}(x)=E_{N}(N(x))$ for all $x \in[0,1]$.

4) If $y \geq x$ when $x \geq e$, and $y \leq x$ when $x \leq e$, then $E_{N}(x) \geq E_{N}(y)$.

Example 3:

$$
\begin{aligned}
& E_{N}(x) \\
& = \begin{cases}\frac{x \log (x)+(1-x) \log (1-x)}{\log (0.5)}, & \text { if } x \neq 0 \text { and } x \neq 1 \\
0, & \text { if } x=0 \text { or } x=1\end{cases}
\end{aligned}
$$

Evidently, in this case, $N(x)=1-x$, and $e=1 / 2$.

Based on Definition 4, we propose the following definition.

Definition 5: A function $H:[0,1]^{2} \rightarrow[0,1]$ is called a bientropic function that is associated with the strong negation $N$, if it satisfies the following conditions.

H1) $H(x, y)=H(y, x)$ for all $x, y \in[0,1]$.

H2) $H(x, y)=0$ if and only if $\{0,1\} \cap\{x, y\} \neq \emptyset$.

H3) $H(x, y)=1$ if and only if $x=y=e$, where $e$ is the equilibrium point of $N$.

H4) $H(x, y)=H(N(x), N(y))$.

H5) $H$ is increasing on $[0, e]^{2}$ and decreasing on $[e, 1]^{2}$. Here, $e \in] 0,1[$ is the only point, such that $N(e)=e$.

Proposition 1: If $E_{N}$ is a normal $E_{N}$-function that is associated with the strong negation $N$, and $C$ is a conjunctive aggregation function with no zero divisors, then

$$
H(x, y)=C\left(E_{N}(x), E_{N}(y)\right) \text { for all } x, y \in[0,1]
$$

is a bientropic function.
Proof: The proof is direct.

Proposition 2: Let $\varphi_{1}$ and $\varphi_{2}$ be two automorphisms of the unit interval; $N$ be any strong negation, such that $N(e)=e$; and $C$ be a conjunctive aggregation function with no zero divisors. Under these conditions

$$
\begin{aligned}
H(x, y)=C\left(\varphi_{1}^{-1}\left(1-\left|\varphi_{2}(x)-\varphi_{2}(N(x))\right|\right)\right. \\
\varphi_{1}^{-1}\left(1-\left|\varphi_{2}(y)-\varphi_{2}(N(y))\right|\right)
\end{aligned}
$$

is a bientropic function.

Proof: The proof is direct.

Example 4: 1) If $\varphi_{1}(x)=x^{\frac{1}{d}}$ with $d \geq 1, \varphi_{2}(x)=x$, and $N(x)=1-x$ for all $x \in[0,1]$, then $E_{N}(x)=\varphi_{1}^{-1}(1-$ $|2 x-1|)=\varphi_{1}^{-1}(1-\max (2 x-1,1-2 x))=\varphi_{1}^{-1}(2 \min (1-$ $x, x))=2^{d}(\min (1-x, x))^{d}$ is Kaufmans' normal $E_{N^{-}}$ function. Under these conditions, if we take $C=\min$, we obtain

$$
H(x, y)=\min \left(2^{d}(\min (1-x, x))^{d}, 2^{d}(\min (1-y, y))^{d}\right) .
$$

2) If we take $\varphi_{1}(x)=1-(1-x)^{d}$ with $d \geq 1, N(x)=1-x$ and $\varphi_{2}(x)=x$ for all $x \in[0,1]$, the normal $E_{N}$-function that we obtain is from Yager, i.e., $E_{N}(x)=\varphi_{1}^{-1}(1-|2 x-1|)=$ $1-|2 x-1|^{d}$. Under these conditions, if we take $C=\min$, we obtain

$$
H(x, y)=\min \left(1-|2 x-1|^{d}, 1-|2 y-1|^{d}\right) .
$$

As mentioned earlier, we seek to establish a connection between generalized bientropic functions and overlap/grouping functions. In order to guarantee desirable properties of the latter, it makes sense to restrict the class of generalized bientropic functions, as in the following definition.

Definition 6: A generalized bientropic function is a mapping, i.e., $G_{E}:[0,1]^{2} \rightarrow[0,1]$, for which there exists $\left.e \in\right] 0,1[$, such that

$\left.G_{E} 1\right) G_{E}(x, y)=G_{E}(y, x)$ for all $x, y \in[0,1]$;

$\left.G_{E} 2\right) G_{E}(x, y)=0$ if $x=1$ or $y=1$;

$\left.G_{E} 3\right) G_{E}(x, y)=1$ if and only if $x=y=e$;

$\left.G_{E} 4\right) G_{E}$ is decreasing in $[e, 1]^{2}$;

$\left.G_{E} 5\right) G_{E}$ is increasing in $[0, e]^{2}$.

Theorem 4: Every continuous bientropic function that is associated with the strong negation $N$ is a generalized bientropic function with $e$ given by the equilibrium point of $N$.

Proof: The proof is direct.

Observe that the converse of Theorem 4 does not hold.

\section{Connection BetweEn Grouping Functions, OverlaP FUnCTIONS, AND GENERALIZED BIENTROPIC FUNCTIONS}

In this section, we connect the concepts of grouping and overlap functions with that of generalized bientropic functions.

First of all observe that the classes of generalized bientropic functions, overlap functions, and grouping functions are disjoint. Note that, if $G_{O}$ is an overlap function, $G_{G}$ is a grouping function and $G_{E}$ is a generalized bientropic function; it follows that $G_{0}(0,1)=G_{E}(0,1)=0$, whereas $G_{G}(0,1)=1$; therefore, a grouping function can never be an overlap function or a generalized bientropic function. On the other side, by definition, there exists $e \in] 0,1\left[\right.$, such that $G_{E}(e, e)=1$ but 


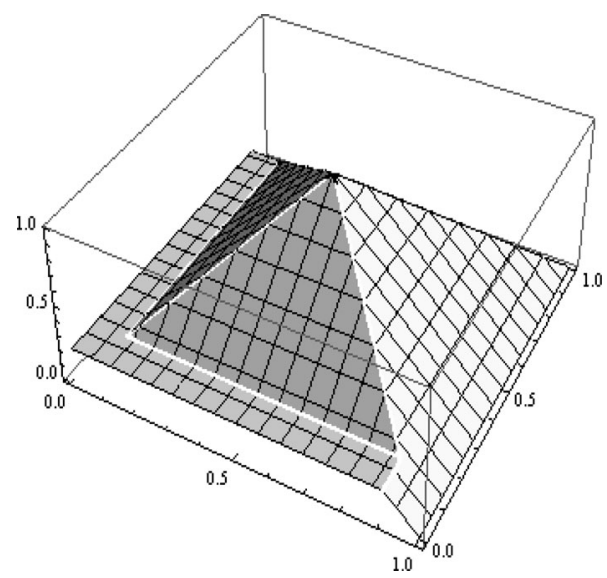

Fig. 1. Example of the generalized bientropic function which satisfies (8).

$G_{0}(e, e) \neq 1$ (since $e \neq 1$ ); therefore, in addition, the classes of overlap functions and generalized bientropic functions are disjoint. Nevertheless, we have the following result.

Proposition 3: Let $G_{E}$ be a generalized bientropic function and $G_{O}$ be an overlap function. Then, the function

$$
P(x, y)=G_{O}\left(G_{E}(x, y), G_{E}(x, y)\right)
$$

is also a generalized bientropic function.

Proof: Continuity is clear since we are dealing with a composite of continuous functions. Symmetry of $P$ is also obvious. Now, if $x=1$ or $y=1$, then $G_{E}(x, y)=$ 0 ; therefore, $P(x, y)=G_{E}(x, y)=0$. Moreover, $P(e, e)=$ $G_{O}\left(G_{E}(e, e), G_{E}(e, e)\right)=G_{O}(1,1)=1$. If $P(x, y)=1=$ $G_{O}\left(G_{E}(x, y), G_{E}(x, y)\right)$, we have $G_{E}(x, y)=1$ if and only if $x=y=e$. Finally, the monotonicity conditions follow from the fact that they hold for $G_{E}$, and $G_{O}$ is monotone nondecreasing.

There is a very close relation between generalized bientropic functions and overlap functions, as the following results show.

Theorem 5: Let $G_{E}$ be a generalized bientropic function, such that

$$
G_{E}(x, y)=0 \text { if and only if } x=1 \text { or } y=1 .
$$

Then, for any strong negation, $N:[0,1] \rightarrow[0,1]$, such that $N(0.5)=0.5$, the mapping

$$
G_{O}(x, y)=G_{E}(N(0.5 \cdot x), N(0.5 \cdot y))
$$

is an overlap function. In addition, reciprocally, let $G_{O}$ be an overlap function, and let $N:[0,1] \rightarrow[0,1]$ be a strong negation. Then, the mapping

$$
G_{E}(x, y)=G_{O}(N(|2 x-1|), N(|2 y-1|))
$$

is a generalized bientropic function.

Proof: It follows from a straight calculation.

Example 5: The mapping (see Fig. 1)

$$
G_{E}(x, y)=\min (2(1-x), 2(1-y), \max (2 x, \epsilon), \max (2 y, \epsilon))
$$

with $\epsilon>0$ and $\epsilon<1 / 4$ is a generalized bientropic function that satisfies (7).
Take $N(x)=1-x$ for all $x \in[0,1]$. Then, from $(8)$

$$
\begin{aligned}
G_{O}(x, y)=\min (x, y, \max (2-x, \epsilon) & \\
\max (2-y, \epsilon)) & =\min (x, y) .
\end{aligned}
$$

Corollary 2: Let $G_{E}$ be a generalized bientropic function, such that

$$
G_{E}(x, y)=0 \text { if and only if } x=1 \text { or } y=1 \text {. }
$$

Then, for any strong negation, i.e., $N:[0,1] \rightarrow[0,1]$, such that $N(0.5)=0.5$, the mapping

$$
G_{G}(x, y)=N\left(G_{E}(N(0.5 \cdot N(x)), N(0.5 \cdot N(y)))\right)
$$

is a grouping function. Reciprocally, let $G_{G}$ be a grouping function. Then, the mapping

$$
G_{E}(x, y)=N\left(G_{G}(|2 x-1|, \mid 2 y-1)\right)
$$

is a generalized bientropic function.

Proof: Direct.

\section{CONNECTION BETWEEN INCOMPARABILITY, OVERLAP, GROUPING, AND GENERALIZED BIENTROPIC FUNCTIONS IN PREFERENCE RELATIONS}

\section{A. Fuzzy Preference Relations}

Let $\mathcal{R} \in \mathcal{F} R(X \times X)$ be a fuzzy preference relation over a set of alternatives, i.e., $X=\left\{x_{1}, \ldots, x_{n}\right\}$ (see [10], [13], [17], [19], [26], and [28]); for each pair of alternatives $x_{i}$ and $x_{j}$, $\mathcal{R}_{i j}=\mathcal{R}\left(x_{i}, x_{j}\right)$ represents a degree of preference of $x_{i}$ over $x_{j}$, namely the degree to which $x_{i}$ is considered as least as good as $x_{j}$ (by definition, $\mathcal{R}_{i i}=1$ or $\mathcal{R}_{i i}$ are not considered).

From a weak preference relation $\mathcal{R}$, in [17] (see also [10] and [30]), the authors derive the following relations.

1) Strict preference, i.e., $\mathcal{P}_{i j}=\mathcal{P}\left(x_{i}, x_{j}\right)$, is a measure of strict preference of $x_{i}$ over $x_{j}$, indicating that $x_{i}$ is (weakly) preferred to $x_{j}$, but $x_{j}$ is not (weakly) preferred to $x_{i}$.

2) Indifference, i.e., $\mathcal{I}_{i j}=\mathcal{I}\left(x_{i}, x_{j}\right)$, is a measure of the simultaneous fulfillment of $\mathcal{R}_{i j}$ and $\mathcal{R}_{j i}$. Roughly speaking, $x_{i}$ and $x_{j}$ are considered equal in the sense that both $x_{i}$ is as good as $x_{j}$ and the other way around.

3) Incomparability, i.e., $\mathcal{J}_{i j}=\mathcal{J}\left(x_{i}, x_{j}\right)$, is a measure of the incomparability of $x_{i}$ and $x_{j}$, which occurs if neither $\mathcal{R}_{i j}$ nor $\mathcal{R}_{j i}$.

Fodor and Roubens axiomatically define these concepts. The three axioms that they present are: independence, monotonicity, and symmetry. In particular, they propose to express the aforementioned relations in terms of t-norms $T_{1}$ and $T$ and a strict negation $N$ :

$$
\begin{aligned}
& \mathcal{P}_{i j}=T_{1}\left(\mathcal{R}_{i j}, N\left(\mathcal{R}_{j i}\right)\right) \\
& \mathcal{I}_{i j}=T\left(\mathcal{R}_{i j}, \mathcal{R}_{j i}\right) \\
& \mathcal{J}_{i j}=T\left(N\left(\mathcal{R}_{i j}\right), N\left(\mathcal{R}_{j i}\right)\right) \text { for all } i, j \in\{1, \ldots, n\} .
\end{aligned}
$$


TABLE I

EXAMPLES OF INDIFFERENCE AND INCOMPARABILITY

\begin{tabular}{cccc}
\hline & $\begin{array}{c}\text { Indifference sense Eq.(15) } \\
G_{O}(x, y)\end{array}$ & Indifference sense Eq.(14) & Incomparability sense Eq.(15) \\
\hline $\min (x, y)$ & Yes & Yes & No \\
$x \cdot y$ & Yes & Yes & No \\
$\min \left(x^{k} y, x y^{k}\right)$ with $\left.k \in\right] 0,1[$ & Yes & No & No \\
$\frac{\sqrt{x y}}{\sqrt{x y}+1-x y}$ & Yes & No & Yes \\
$1-(\max (x, y))^{k}$ with $k>0$ & No & No & Yes \\
$\frac{\sqrt{(1-x)(1-y)}}{\max (x, y)+\sqrt{(1-x)(1-y)}}$ & No & No & \\
\hline
\end{tabular}

Note that, according to the authors' axiomatization, the expressions in (14) are not the only possibilities, even more since associativity is not required; in fact, every monotone, symmetric bivariate function would do, in principle. These considerations lead us to consider the overlap and grouping of two (weak) preference degrees as alternative operations for deriving, respectively, a degree of indifference and a degree of incomparability since they also satisfy the three axioms of Fodor and Roubens. That is, we propose to define indifference and incomparability by

$$
\begin{aligned}
& \mathcal{I}_{i j}=G_{O}\left(\mathcal{R}_{i j}, \mathcal{R}_{j i}\right) \\
& \mathcal{J}_{i j}=1-G_{G}\left(\mathcal{R}_{i j}, \mathcal{R}_{j i}\right) .
\end{aligned}
$$

Observe that expressions in (15) fulfill the independence, monotonicity, and symmetry axioms of Fodor and Roubens, but they are not forcibly obtained from t-norms, since we have not imposed associativity in the definition of overlap functions. There exist hence indifferences in the sense of (14) that are not indifferences in our sense; it is enough to consider any t-norm with divisors of zero. Conversely, there exist indifferences in our sense that are not indifferences in the sense of (14), just by considering any overlap function that is not a t-norm. See Table I.

Taking into account the constructions in Sections III-V, it turns out that the concepts of indifference (overlap) and incomparability (negation of grouping) can be written in terms of generalized bientropic functions. More concretely, we can show the following result.

Corollary 3: Let $\mathcal{R} \in \mathcal{F} R(X \times X), N$ be a strong negation, such that $N(0.5)=0.5$, and let $G_{E}$ be a generalized bientropic function, such that

$$
G_{E}(x, y)=0 \text { if and only if } x=1 \text { or } y=1 .
$$

Then, if the overlap function $G_{O}$ that is associated with $G_{E}$ by Theorem 5 is such that $G_{O}(x, 1-x) \leq 1 / 2$ for all $x \in[0,1]$, the following holds.

1) $\mathcal{I}_{i j}=G_{O}\left(\mathcal{R}_{i j}, \mathcal{R}_{j i}\right)=G_{E}\left(1-\frac{\mathcal{R}_{i j}}{2}, 1-\frac{\mathcal{R}_{j i}}{2}\right)$.

2) $\mathcal{J}_{i j}=1-G_{G}\left(\mathcal{R}_{i j}, \mathcal{R}_{j i}\right)=G_{E}\left(\frac{1+\mathcal{R}_{i j}}{2}, \frac{1+\mathcal{R}_{j i}}{2}\right)$.

Proof: Just recall Corollary 5.

Note that, from the condition, i.e., $G_{O}(x, 1-x) \leq 1 / 2$, that is imposed on the overlap function, it follows that $\mathcal{I}_{i j}+\mathcal{J}_{i j} \leq 1$.

\section{B. Grouping and Overlap Functions in Fuzzy Decision Making Using General Relations}

In this section, we are going to consider the decision-making problem in a setting in which an expert expresses his or her preferences over a set of alternatives. It is assumed that the set of alternatives is finite:

$$
X=\left\{x_{1}, \ldots, x_{n}\right\} \text { with } n \geq 2 .
$$

Depending on the nature of alternatives and of the knowledge of the experts about those alternatives, preferences can be expressed in different ways. In this paper, we consider that this expression is done using fuzzy sets (see [8]).

We assume that the starting point is a fuzzy binary preference relation, which is not necessarily normalized:

$$
\mathcal{R}=\left(\begin{array}{cccc}
- & R_{12} & \ldots & R_{1 n} \\
R_{21} & - & \ldots & R_{2 n} \\
\ldots & \ldots & - & \ldots \\
R_{n 1} & \ldots & \ldots & -
\end{array}\right)
$$

We want to act upon the fuzzy relation $\mathcal{R}$ to obtain an ordering of the alternatives that allows us to make a decision (to choose one of them). Moreover, we want to penalize

1) values of preferences that are close to 0.5 , i.e., situations in which an expert is not sure of whether he/she prefers an alternative against another;

2) possible indifference of the expert in his/her preference of one alternative against another;

3) possible incomparability between two alternatives.

These considerations have led us to propose an algorithm for the construction of the ranking of preference of alternatives, such that it minimizes an expression that is associated with each alternative $x_{i}$ and made up of three terms: the generalized bientropy that is associated with $\mathcal{P}_{i j}$, the indifference between $R_{i j}$ and $R_{j i}$, and the incomparability between both.

Given a fuzzy preference relation, i.e., $\mathcal{R} \in \mathcal{F} S(X \times X)$, we build the strict preference relation $\mathcal{P}$ that is associated with $\mathcal{R}$ by means of the expression that is proposed by Fodor and Roubens as follows:

$$
\mathcal{P}_{i j}=T_{L}\left(\mathcal{R}_{i j}, N\left(\mathcal{R}_{j i}\right)\right)
$$

with $T_{L}(x, y)=\max (0, x+y-1)$ (Eukasiewicz t-norm) and $N$ being the standard negation. It is easy to see that in this setting the strict preference relation is given by

$$
\mathcal{P}_{i j}= \begin{cases}R_{i j}-R_{j i}, & \text { if } R_{i j}>R_{j i} \\ 0, & \text { otherwise. }\end{cases}
$$




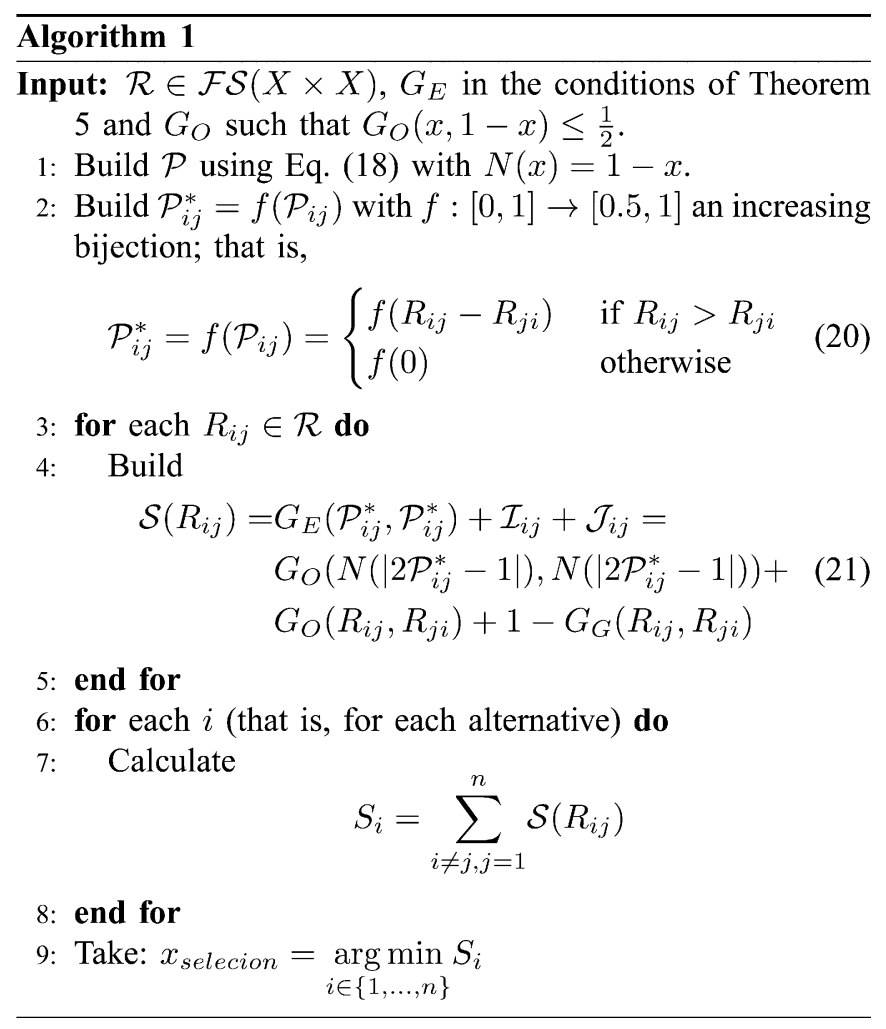

The algorithm that we propose to choose the best alternative is the following (see Algorithm 1).

\section{Justification of Steps in Algorithm 1}

1) The transformation from $\mathcal{P}_{i j}$ to $\mathcal{P}_{i j}^{*}$ in Step 2 allows us to ensure that $0.5 \leq \mathcal{P}_{i j}^{*} \leq 1$. Then, by Theorem 5 , we have

$$
G_{E}\left(\mathcal{P}_{i j}^{*}, \mathcal{P}_{i j}^{*}\right)=0 \text { if and only if } \mathcal{P}_{i j}^{*}=1 .
$$

That is, the minimum of the generalized bientropic function is obtained for $\mathcal{P}_{i j}^{*}=1$. Moreover, we do not consider the case, i.e., $\mathcal{P}_{i j}^{*}=0$. Since $f$ is an increasing bijection, if $\mathcal{P}_{i j}^{*}=1$, from (20), it follows that $R_{i j}=1$ and that $R_{j i}=0$.

2) In $G_{E}$, we take both arguments equal: $G_{E}\left(\mathcal{P}_{i j}^{*}, \mathcal{P}_{i j}^{*}\right)$. This is so since in case $R_{i j}<R_{j i}$, it holds that $G_{E}\left(\mathcal{P}_{i j}^{*}, \mathcal{P}_{i j}^{*}\right)=$ 1 , i.e., we penalize alternative $x_{i}$ if the preference of $x_{i}$ over $x_{j}$ is less than the preference of $x_{j}$ over $x_{i}$.

3) The minimum of

$$
S_{i}=\sum_{j=1}^{n} \mathcal{S}\left(R_{i j}\right)=\sum_{j=1}^{n} G_{E}\left(\mathcal{P}_{i j}^{*}, \mathcal{P}_{i j}^{*}\right)+\mathcal{I}_{i j}+\mathcal{J}_{i j}
$$

is attained when all the summands are minimal, i.e., when $G_{E}\left(\mathcal{P}_{i j}^{*}, \mathcal{P}_{i j}^{*}\right)$, indifference and incomparability between $R_{i j}$ and $R_{j i}$ are minimal.

4) In step 5, we select the alternative that is associated with the row of the relation $\mathcal{R}$, such that each of its elements is closer to 1 , and such that indifference and incomparability between the considered element in the relation and its opposite with respect to the main diagonal are also minimal.

Proposition 4: If, in Steps 2 and 3 of Algorithm 1, we take $f(x)=\frac{1}{2} x+\frac{1}{2}$ and $G_{E}$ that is given by (9), respectively, then $G_{E}\left(\mathcal{P}_{i j}^{*}, \mathcal{P}_{i j}^{*}\right)=$

$\begin{cases}G_{O}\left(N\left(R_{i j}-R_{j i}\right), N\left(R_{i j}-R_{j i}\right)\right), & \text { if } R_{i j}>R_{j i} \\ 1, & \text { otherwise. }\end{cases}$

Proof: The proof is direct.

Proposition 5: The following items hold.

1)

$$
\begin{aligned}
& \mathcal{I}_{i j}+\mathcal{J}_{i j}= G_{O}\left(R_{i j}, R_{j i}\right)+1-G_{G}\left(R_{i j}, R_{j i}\right)=0 \\
& \quad \text { if and only if } \\
& \\
&\left\{\begin{array}{l}
R_{i j}=0 \text { and } R_{j i}=1 \\
\text { or } \\
R_{i j}=1 \text { and } R_{j i}=0 .
\end{array}\right.
\end{aligned}
$$

2) If $R_{i j}=R_{j i}=1$, then $\mathcal{I}_{i j}+\mathcal{J}_{i j}=G_{O}\left(R_{i j}, R_{j i}\right)+1-$ $G_{G}\left(R_{i j}, R_{j i}\right)=1$.

3) If $R_{i j}=R_{j i}=0$, then $\mathcal{I}_{i j}+\mathcal{J}_{i j}=G_{O}\left(R_{i j}, R_{j i}\right)+1-$ $G_{G}\left(R_{i j}, R_{j i}\right)=1$.

Proof: The proof is direct.

Note that, from Item 1 , in Proposition 5 , if $\mathcal{R}$ is a crisp relation, such that for every $i \neq j$, we have $R_{i j}+R_{j i}=1$, then the sum of the indifference and the incomparability is equal to zero. However, from Items 2 and 3, we have that if the relation is crisp and nonadditive, then the sum of the indifference and the incomparability for the elements not fulfilling the additivity property is equal to 1 .

Corollary 4: In the setting of Algorithm 1, the following items hold.

1) If $R_{i j}=1$ and $R_{j i}=0$, then $\mathcal{S}\left(R_{i j}\right)=0+0+0=0$.

2) If $R_{i j}=0$ and $R_{j i}=1$, then $\mathcal{S}\left(R_{i j}\right)=1+0+0=1$.

Proof: The proof is direct.

Note that Items 1 and 2 of Corollary 4 correspond to the ideal (crisp) case, i.e., our algorithm makes sense when reduced to the crisp case.

Corollary 5: In the setting of Corollary 3

$$
\begin{aligned}
\mathcal{S}\left(R_{i j}\right)= & G_{E}\left(\mathcal{P}_{i j}^{*}, \mathcal{P}_{i j}^{*}\right)+G_{E}\left(1-\frac{\mathcal{R}_{i j}}{2}, 1-\frac{\mathcal{R}_{j i}}{2}\right) \\
& +G_{E}\left(\frac{1+\mathcal{R}_{i j}}{2}, \frac{1+\mathcal{R}_{j i}}{2}\right) .
\end{aligned}
$$

This corollary shows that solving a decision-making problem with our algorithm is equivalent to minimizing a function of the generalized bientropy. This fact fully agrees with what happens in most of the image thresholding fuzzy algorithms in image processing [20].

Proof: The proof is direct.

Proposition 6: For the elements of the relation $\mathcal{R} \in \mathcal{F} \mathcal{R}(X \times$ $X$ ), such that $R_{i j}+R_{j i}=1$, with the constructions from (15) and $N(x)=1-x$, we have that $\mathcal{I}_{i j}=\mathcal{J}_{i j}$.

Proof: The proof is direct. 
In fact, we can settle a stronger result for reciprocal additive relations that links our developments with the concept of a weak ignorance function that is given in [32] and [33].

Theorem 6: If $\mathcal{R} \in \mathcal{F} R(X \times X)$ is the reciprocal additive and $G_{O}(0.5,0.5)=0.5$, then the mapping

$$
g\left(R_{i j}\right)=\mathcal{I}_{i j}+\mathcal{J}_{i j}=2 G_{O}\left(R_{i j}, 1-R_{i j}\right)
$$

satisfies the following items.

1) $g\left(R_{i j}\right)=g\left(1-R_{i j}\right)$.

2) $g\left(R_{i j}\right)=0$ if and only if $R_{i j}=0$ or $R_{i j}=1$.

3) if $R_{i j}=R_{j i}=\frac{1}{2}$, then $g\left(R_{i j}\right)=1$.

Proof: The proof is direct.

We should stress that in the setting of Theorem 6, the properties of the function $g\left(R_{i j}\right)$ coincide with those demanded for the weak ignorance function (see [5], [32], and [33]) evaluated in $R_{i j}$. Such a function must be understood as the ignorance that is associated with the value $R_{i j}$, which is provided by the expert. It must not be confused with the idea of ignorance that is associated with an event as considered in possibility theory [14]. In our case, it is a function that associates with each of the values that are given by the expert a degree of ignorance that only depends of the value itself. That is, we are not speaking of ignorance that is associated with a proposition.

The following example shows how our algorithm penalizes the preference values close to 0.5 .

Example 6: Let $X=\left\{x_{1}, x_{2}, x_{3}, x_{4}, x_{5}\right\}$ be a set of alternatives. Consider the fuzzy preference relation

$$
\mathcal{R}=\left(\begin{array}{cccccc} 
& x_{1} & x_{2} & x_{3} & x_{4} & x_{5} \\
x_{1} & - & 0.82 & 0.4 & 0.26 & 0.72 \\
x_{2} & 0.18 & - & 0.19 & 0.53 & 0.46 \\
x_{3} & 0.78 & 0.43 & - & 0.53 & 0.5 \\
x_{4} & 0.6 & 0.47 & 0.58 & - & 0.59 \\
x_{5} & 0.43 & 0.28 & 0.15 & 0.08 & -
\end{array}\right) .
$$

We take $f(x)=\frac{1}{2} x+\frac{1}{2}, N(x)=1-x$, and $G_{E}\left(\mathcal{P}_{i j}^{*}, \mathcal{P}_{i j}^{*}\right)=$ $\left(1-\left(R_{i j}-R_{j i}\right)\right) \cdot\left(1-\left(R_{i j}-R_{j i}\right)\right)$. We analyze the following cases.

1) $G_{O}(x, y)=\min (x, y)$. Then, we have $\mathcal{I}_{i j}=\min \left(R_{i j}\right.$, $\left.R_{j i}\right)$ and $\mathcal{J}_{i j}=\min \left(1-R_{i j}, 1-R_{j i}\right)$. From Step 5 in Algorithm 1

$$
x_{1}=\underset{i \in\{1, \ldots, 5\}}{\arg \min }\left\{x_{1}=3.525, x_{2}=4.32, x_{3}=4.47\right.
$$

$$
\left.x_{4}=4.56, x_{5}=4.005\right\} .
$$

2) $G_{O}(x, y)=\sqrt{x \cdot y}$. Then, $\mathcal{I}_{i j}=\sqrt{R_{i j} \cdot R_{j i}}$ and $\mathcal{J}_{i j}=$ $\sqrt{\left(1-R_{i j}\right) \cdot\left(1-R_{j i}\right)}$. From Step 5 in Algorithm 1

$$
\begin{array}{r}
x_{3}=\underset{i \in\{1, \ldots, 5\}}{\arg \min }\left\{x_{1}=4.7602, x_{2}=5.1543, x_{3}=5.3017\right. \\
\left.x_{4}=5.2874, x_{5}=5.0305\right\} .
\end{array}
$$

3) Voting method [15]

$$
\begin{array}{r}
x_{3}=x_{4}=\underset{i \in\{1, \ldots, 5\}}{\arg \min }\left\{x_{1}=2.2, x_{2}=1.36, x_{3}=2.24\right. \\
\left.x_{4}=2.26, x_{5}=0.94\right\} .
\end{array}
$$

4) Nondominance method [27]

$$
\begin{array}{r}
x_{3}=\underset{i \in\{1, \ldots, 5\}}{\arg \min }\left\{x_{1}=0.6047, x_{2}=0.36, x_{3}=0.9550\right. \\
\left.x_{4}=0.94, x_{5}=0.2388\right\} .
\end{array}
$$

Remark:

1) Note that, in Example 6, the voting method chooses the fourth alternative, and the nondominance method chooses the third one. However, our methods pick up the first alternative. The reason for this is that in the fuzzy preference relations, the values in the third and the fourth rows are close to 0.5 ; therefore, we penalize both.

2) Note that for the first two cases, it holds that $\mathcal{I}_{i j}+\mathcal{J}_{i j} \leq 1$.

\section{Learning Fuzzy Preferences From Data}

The idea of using fuzzy preference relations for representing different types of uncertainty in machine learning, or more specifically, in pairwise classification, was recently put forward in [22]. In this paper, it is settled that given an instance $x$ to be classified and two candidate classes $C_{i}$ and $C_{j}$, classification knowledge is expressed in terms of a degree of strict preference for $C_{i}$, a degree of strict preference for $C_{j}$, the so-called degree of conflict between $C_{i}$ and $C_{j}$, and the so-called degree of ignorance between classes. Roughly speaking, this concept of conflict in classification corresponds to the concept of indifference in decision making; a positive degree of conflict suggests that the data provide evidence in favor of both classes $C_{i}$ and $C_{j}$, simultaneously. This concept of ignorance between classes, on the other hand, corresponds to the concept of incomparability; a positive degree of ignorance suggests that the data neither provide evidence in favor of $C_{i}$ nor in favor of $C_{j}$. In this section, we consider a generic approach that allows one to derive such degrees from estimated lower and upper bounds on the conditional probability of $C_{i}$ given $C_{i}$ or $C_{j}$.

Consider two classes, i.e., $C_{i}$ and $C_{j}$, and suppose incomplete probabilistic information to be given, namely information about the probability of $C_{i}$ in the form of an interval $[a, b] \subset[0,1]$; more precisely, this means that

$$
a \leq \operatorname{Pr}\left(q \in C_{i} \mid q \in C_{i} \cup C_{j}\right) \leq b .
$$

In this case, the weak preference for $C_{i}$ and $C_{j}$, respectively, should of course depend on $a$ and $b$, and a possible definition is

$$
\mathcal{R}_{i j}=\frac{a}{\max (a, 1-b, 1 / 2)}, \quad \mathcal{R}_{j i}=\frac{1-b}{\max (a, 1-b, 1 / 2)} .
$$

Regarding the modeling of indifference and incomparability in terms of (15), the following cases can be distinguished.

1a) $a+b \leq 1$, and $a \leq b<1 / 2$ (interval $[a, b]$ to the left of $1 / 2)$.

1b) $a+b \leq 1$, and $a<1 / 2<b$ (interval $[a, b]$ overlapping $1 / 2$ in favor of $C_{j}$ ).

2a) $a+b \geq 1$, and $a<1 / 2<b$ (interval $[a, b]$ overlapping $1 / 2$ in favor of $C_{i}$ ).

2b) $a+b \geq 1$, and $1 / 2<a \leq b$ (interval $[a, b]$ to the right of $1 / 2)$. 
For the weak preferences and, consequently, for the indifference and incomparability relation, we then have

1a) $\mathcal{R}_{i j}<1, \mathcal{R}_{j i}=1, \mathcal{I}_{i j} \geq 0, \mathcal{J}_{i j}=0$;

1b) $\mathcal{R}_{i j}<1, \mathcal{R}_{j i}<1, \mathcal{I}_{i j} \geq 0, \mathcal{J}_{i j} \geq 0$;

2a) $\mathcal{R}_{i j}<1, \mathcal{R}_{j i}<1, \mathcal{I}_{i j} \geq 0, \mathcal{J}_{i j} \geq 0$,

2b) $\mathcal{R}_{i j}=1, \mathcal{R}_{j i}<1, \mathcal{I}_{i j} \geq 0, \mathcal{J}_{i j}=0$.

Roughly speaking, the degree of indifference will depend on how close the interval $[a, b]$ is to the boundary points. The closer it is to 0 or to 1 , the smaller the indifference. Incomparability increases with the length of the interval but is strictly positive only in the cases, where the interval overlaps the middle point $1 / 2$. The interpretation is that, as long as the interval is either fully to the left of $1 / 2$ or fully to the right, there is no doubt about the best decision, since in the first case, one should decide in favor of $C_{j}$, and in the second case, in favor of $C_{i}$.

More concretely, let $G_{O}(x, y)=\sqrt{x y} /(\sqrt{x y}+1-x y)$ and $G_{G}(x, y)=1-G_{O}(1-x, 1-y)$. We then obtain $\mathcal{I}_{i j}=0$ and $\mathcal{J}_{i j}=1$ for $[a, b]=[0,1]$ (the case of maximal incomparability), $\mathcal{I}_{i j}=1$ and $\mathcal{J}_{i j}=0$ for $[a, b]=[1 / 2,1 / 2]$ (the case of maximal indifference), $\mathcal{I}_{i j}=0.55$ and $\mathcal{J}_{i j}=0.27$ for $[a, b]=$ $[1 / 3,2 / 3]$ (interval overlapping $1 / 2$ leads to partial indifference and ignorance), $\mathcal{I}_{i j}=0.5$ and $\mathcal{J}_{i j}=0$ for $[a, b]=[1 / 4,1 / 3]$ (interval to the left of $1 / 2$, no incomparability but still some degree of indifference).

Based on this preference structure, predictions can be derived using Algorithm 1 (or the decision rule given in [21] and [22]). As future work, we plan to elaborate on this alternative more closely. In particular, we are interested in the idea of adapting the overlap and grouping functions to the problem at hand, i.e., to learn a most suitable bientropic function from the data. Roughly, the idea is to select one such function from a parametrized family to maximize the predictive performance of the classifier. In this regard, an important advantage of grouping and overlap functions (in comparison to t-norms and t-conorms) deserves mentioning, namely, the convexity of these classes of functions. From a learning point of view, this is a very appealing property, e.g., as it allows one to represent an aggregation function as a convex combination of a class of basis (aggregation) functions. In fact, many machine learning methods make use of this kind of representation.

\section{OPEN PROBLEMS}

In this section, we present a set of interesting open problems that we seek to address in future work.

1) In Algorithm 1, we have introduced the following expression:

$$
\mathcal{S}\left(R_{i j}\right)=G_{E}\left(\mathcal{P}_{i j}^{*}, \mathcal{P}_{i j}^{*}\right)+\mathcal{I}_{i j}+\mathcal{J}_{i j}
$$

The problem that we pose is to analyze the behavior of the algorithm when we take

$$
\mathcal{S}\left(R_{i j}\right)=G_{E}\left(\mathcal{P}_{i j}^{*}, \mathcal{P}_{i j}^{*}\right)+\alpha \mathcal{I}_{i j}+\beta \mathcal{J}_{i j}
$$

with $\alpha$ and $\beta$ being two positive real parameters.

2) Since convex combinations of overlap functions are again overlap functions, we propose to study the advantages of using such combinations in the aggregation phase of multicriteria decision-making problems.

3) Make an exhaustive comparative study of the advantages/disadvantages of Algorithm 1 against other methods that are developed for the exploitation phase in multiexpert decision-making problems, apart from those that are already considered in Section VI after Algorithm 1.

\section{CONCLUSION}

The main contributions and results of this paper can be summarized as follows.

1) The concept of grouping functions, which is a specific type of aggregation function, has been introduced and studied. Roughly speaking, a grouping function measures the degree of excedance of the support of two (mutually exclusive) decision alternatives. In specific cases, its negation can be used to model the notion of incomparability in fuzzy preference structures.

2) Grouping functions complement the existing concept of overlap functions. Because of their properties, the latter naturally qualify for the measurement of the degree of indifference between decision alternatives.

3) Generalized bientropic functions have been introduced as a unifying concept. These functions can be seen as an extension of an entropy function. As such, they provide a measure of noninformation or, say, of the lack of knowledge in connection with the selection between two alternatives.

4) The concepts of overlap and grouping can be built, under suitable conditions, by means of generalized entropy functions.

5) We have advocated the use of grouping and overlap functions in the context of decision making. We propose an algorithm for the exploitation phase that penalizes values close to 0.5 that are given by experts when evaluating their preference of one alternative over another. That is, it penalizes those cases in which experts are not sure of their preferences.

\section{REFERENCES}

[1] J. Barzilai, "Consistency measures for pairwise comparison matrices," $J$. Multi-Criteria Decision. Anal., vol. 7, pp. 123-132, 1998.

[2] G. Beliakov, A. Pradera, and T. Calvo, "What is an aggregation function," in Aggregation Functions: A Guide for Practitioners (Studies in Fuzziness and Soft Computing Series 221), G. Beliakov, A. Pradera, and T. Calvo, Eds. New York: Springer-Verlag, 2007, pp. 1-37.

[3] H. Bustince, P. Burillo, and F. Soria, "Automorphisms, negations and implication operators," Fuzzy Sets Syst., vol. 134, no. 2, pp. 209-229, 2003.

[4] H. Bustince, E. Barrenechea, and M. Pagola, "Restricted equivalence functions," Fuzzy Sets Syst., vol. 157, no. 17, pp. 2333-2346, 2006.

[5] H. Bustince, M. Pagola, E. Barrenechea, J. Fernandez, P. Melo-Pinto, P. Couto, H. R. Tizhoosh, and J. Montero, "Ignorance functions: An application to the calculation of the threshold in prostate ultrasound images," Fuzzy Sets Syst., vol. 161, no. 1, pp. 20-36, 2010.

[6] H. Bustince, J. Fernandez, R. Mesiar, J. Montero, and R. Orduna, "Overlap functions," Nonlinear Anal., Theory, Methods Appl., vol. 72, nos. 3-4, pp. 1488-1499, 2010.

[7] H. Bustince, E. Barrenechea, and M. Pagola, "Relationship between restricted dissimilarity functions, restricted equivalence functions and normal E-N-functions: Image thresholding invariant," Pattern Recog. Lett., vol. 29 , no. 4 , pp. 525-536, 2008. 
[8] F. Chiclana, F. Herrera, and E. Herrera-Viedma, "Integrating three representation models in fuzzy multipurpose decision making based on fuzzy preference relations," Fuzzy Sets Syst., vol. 97, no. 1, pp. 33-48, 1998.

[9] F. Chiclana, F. Herrera, and E. Herrera-Viedma, "Integrating multiplicative preference relations in a multipurpose decision-making model based on fuzzy preference relations," Fuzzy Sets Syst., vol. 122, pp. 277-291, 2001.

[10] B. De Baets, B. Van de Walle, and E. Kerre, "Fuzzy preference structures without incomparability," Fuzzy Sets Syst., vol. 76, no. 3, pp. 333-348, 1995.

[11] D.-F. Li, "TOPSIS-based nonlinear-programming methodology for multiattribute decision making with interval-valued intuitionistic fuzzy sets," IEEE Trans. Fuzzy Syst., vol. 18, no. 2, pp. 299-311, Apr. 2010.

[12] D.-F. Li, "Mathematical-programming approach to matrix games with payoffs represented by Atanassov's interval-valued intuitionistic fuzzy sets," IEEE Trans. Fuzzy Syst., vol. 18, no. 6, pp. 1112-1128, Dec. 2010.

[13] W. Dongrui and J. M. Mendel, "Computing with words for hierarchical decision making applied to evaluating a weapon system," IEEE Trans. Fuzzy Syst., vol. 18, no. 3, pp. 441-460, Jun. 2010.

[14] D. Dubois and H. Prade, Possibility Theory. New York: Plenum, 1988.

[15] M. Dummett, A Voting Procedures. $\quad$ Oxford, U.K.: Clarendon, 1984.

[16] M. Fedrizzi and S. Giove, "Incomplete pairwise comparison and consistency optimization," Eur. J. Oper. Res., vol. 183, pp. 303-313, 2007.

[17] J. Fodor and M. Roubens, "Fuzzy preference modelling multicriteria decision support," in Theory and Decision Library, New York: Kluwer, 1994.

[18] M. Grabisch, J.-L. Marichal, R. Mesiar, and E. Pap, Aggregation Functions. Cambridge, MA: Cambridge Univ. Press, 2009.

[19] F. Herrera, L. Martínez, and P. J. Sánchez, "Managing non-homogeneous information in group decision making," Eur. J. Oper. Res., vol. 166, no. 1, pp. 115-132, 2005.

[20] L. K. Huang and M. J. Wang, "Image thresholding by minimizing the measure of fuzziness," Pattern Recog., vol. 28, no. 1, pp. 41-51, 1995.

[21] J. C. Hühn and E. Hüllermeier, "FR3: A fuzzy rule learner for inducing reliable classifiers," IEEE Trans. Fuzzy Syst., vol. 17, no. 1, pp. 138-149, Feb. 2009

[22] E. Hüllermeier and K. Brinker, "Learning valued preference structures for solving classification problems," Fuzzy Sets Syst., vol. 159, no. 18 , pp. 337-2352, 2008.

[23] J. Kacprzyk, "Group decision making with a fuzzy linguistic majority," Fuzzy Sets Syst., vol. 18, pp. 105-118, 1986.

[24] R. L. Keene and H. Raiffa, Decisions With Multiple ObjectivesPreferences and Value Tradeoffs. New York: Wiley, 1976.

[25] E. P. Klement, R. Mesiar, and E. Pap, Triangular Norms, Trends in Logic (Studia Logica Library Series 8). Dordrecht, The Netherlands: Kluwer, 2000.

[26] J. M. Mendel, J. Lawry, and L. A. Zadeh, "Foreword to the special section on computing with words," IEEE Trans. Fuzzy Syst., vol. 18, no. 3, pp. 437-440, Jun. 2010.

[27] S. A. Orlovsky, "Decision-making with a fuzzy preference relation," Fuzzy Sets Syst., vol. 1, no. 3, pp. 155-167, 1978.

[28] S. V. Ovchinnikov and M. Roubens, "On strict preference relations," Fuzzy Sets Syst., vol. 43, pp. 319-326, 1991.

[29] W. Pedrycz and M. Song, "Analytic hierarchy process (AHP) in group decision making and its optimization with an allocation of information granularity," IEEE Trans. Fuzzy Syst., vol. 19, no. 3, pp. 527-539, Jun. 2011.

[30] M. Roubens and P. Vincke, Preference Modellin (Lecture Notes in Economics and Mathematical Systems Series 250). Berlin, Germany: Springer-Verlag, 1985.

[31] T. L. Saaty, The Analytical Hierarchy Process. New York: McGrawHill, 1980

[32] J. A. Sanz, A. Fernandez, H. Bustince, and F. Herrera, "Improving the performance of fuzzy rule-based classification systems with interval-valued fuzzy sets and genetic amplitude tuning," Inf. Sci., vol. 180, no. 19, pp. 3674-3685, 2010.

[33] J. A. Sanz, A. Fernandez, H. Bustince, and F. Herrera, "A genetic tuning to improve the performance of fuzzy rule-based classification systems with interval-valued fuzzy sets: Degree of ignorance and lateral position," Int. J. Approx. Reason. vol. 52, no. 6, pp. 751-766, 2011.

[34] E. Trillas, "Sobre funciones de negación en la teoría de conjuntos difusos," Stochastica, III-1, pp. 47-59, 1979 (in Spanish) [Reprinted (English version) in Advances of Fuzzy Logic, S. Barro et al., Eds. Univ. de Santiago de Compostela, 1998, pp. 31-43].

[35] Z. Xu and R. R. Yager, "Power-geometric operators and their use in group decision making," IEEE Trans. Fuzzy Syst., vol. 18, no. 1, pp. 94-105, Feb. 2010 .

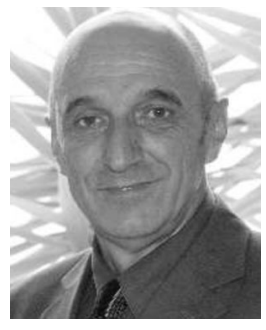

Humberto Bustince (M'08) received the Ph.D. degree in mathematics from the Public University of Navarra, Pamplona, Spain, in 1994.

He is currently a Full Professor with the Department of Automatics and Computation, Public University of Navarra. He is the author of more than 65 published original articles and is involved in teaching artificial intelligence for students of computer sciences. His research interests include fuzzy logic theory, extensions of fuzzy sets (type-2 fuzzy sets, interval-valued fuzzy sets, Atanassovs intuitionistic fuzzy sets), fuzzy measures, aggregation functions, and fuzzy techniques for image processing.

Dr. Bustince is a board member of the European Society for Fuzzy Logic and Technology. He is the Editor-in-Chief of the Mathware and Soft Computing Magazine.

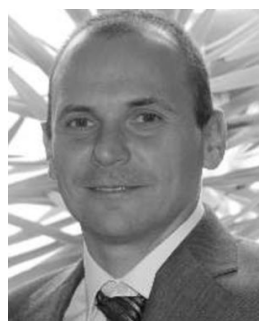

Miguel Pagola received the M.Sc. degree in industrial engineering and the Ph.D. degree, both from the Public University of Navarra (UPNa), Pamplona, Spain, in 2000 and 2008, respectively.

$\mathrm{He}$ is currently an Associate Lecturer with the Department of Automatics and Computation, UPNa. $\mathrm{He}$ is the author of more than 20 published original articles in the Web of Science and is involved in teaching artificial intelligence for students of computer sciences. His research interests include fuzzy techniques for image processing, fuzzy set theory, medical image segmentation, and medical data mining.

Dr. Pagola is the member of the European Society for Fuzzy Logic and Technology.

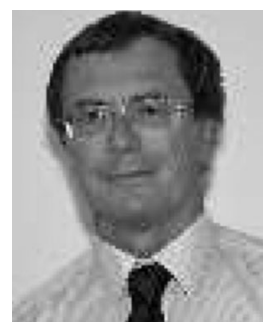

Radko Mesiar received the graduate degree in mathemathics and the Ph.D. degree from the Faculty of Mathematics and Physics, Comenius University, in 1974 and 1979, respectively, with a Ph.D. dissertation on "subadditive martingale processes."

$\mathrm{He}$ is currently the Head of the Department of Mathematics, Faculty of Civil Engineering, Slovak University of Technology (STU), Bratislava, Slovakia. Since 1978, he has been a Member of the Department of Mathematics, Faculty of Civil Engineering, STU, and has been the Doctor of Science since 1996 (with the Czech Academy of Sciences, Prague, Czech Republic). He has been an Associate Professor since 1983 and a Full Professor since 1998. He has been a Fellow of the Institute of Information and Automation, Czech Academy of Sciences, since 1995, and of the Institute for Research and Applications of Fuzzy Modeling, University of Ostrava, Czech Republic, since 2006. He is the co-author of two scientific monographs, i.e., Triangular Norms (Kluwer, 2000), Aggregation Functions (Cambridge University Press, 2009), and five edited volumes. He is the author of more than 200 papers in WOS in journals, e.g., Fuzzy Sets and Systems, Information Sciences, the IEEE TRANSACTIONS ON FUZZY SYSTEMS, THE International Journal of General Systems, the Journal of Mathematical Analysis, the International Journal of Uncertainty, Fuzziness and Knowledge-Based Systems, Kybernetika, the European Journal of Operational Research, Applied Mathematics Letters, Nonlinear Analysis, etc.

Dr. Mesiar is the Founder and organizer of the Fuzzy Set Theory and Applications and Aggregation Operators Conferences. 


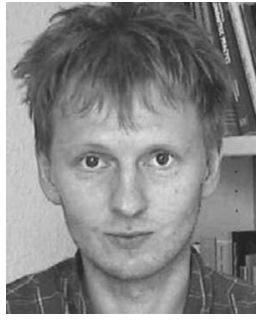

Eyke Hüllermeier received the M.Sc. degrees in mathematics and business computing, the Ph.D. degree in computer science, and a Habilitation degree, all from the University of Paderborn, Germany.

$\mathrm{He}$ is currently a Full Professor with the Department of Mathematics and Computer Science, Marburg University, Marburg, Germany. He is the author/co-author of numerous published research papers on the topics of his research interests in respected journals and major international conferences. His research interests include machine learning and data mining, fuzzy set theory, uncertainty and approximate reasoning, and applications in bioinformatics.

Dr. Hüllermeier is the member of the IEEE Computational Intelligence Society (CIS) and a board member of the European Society for Fuzzy Logic and Technology (EUSFLAT). He is the Co-Editor-in-Chief of Fuzzy Sets and Systems and an editorial board member of several other journals. Moreover, he is the Coordinator of the EUSFLAT working group on learning and data mining and the Head of the IEEE CIS Task Force on machine learning.

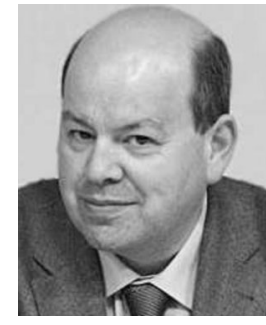

Francisco Herrera (M'10) received the M.Sc. and Ph.D. degrees in mathematics in 1988 and 1991, respectively, both from the University of Granada, Granada, Spain

He is currently a Professor with the Department of Computer Science and Artificial Intelligence, University of Granada. He has authored/co-authored more than 200 published papers in various international journals. He is the coauthor of the book Genetic Fuzzy Systems: Evolutionary Tuning and Learning of Fuzzy Knowledge Bases (World Scientific, 2001). His current research interests include computing with words and decision making, data mining, bibliometrics, data preparation, instance selection, fuzzy rule-based systems, genetic fuzzy systems, knowledge extraction based on evolutionary algorithms, memetic algorithms, and genetic algorithms.

Dr. Herrera is currently the Editor-in-Chief of the international journal Progress in Artificial Intelligence (Springer) and the Area Editor of the Journal Soft Computing (area of evolutionary and bioinspired algorithms) and the International Journal of Computational Intelligence Systems (area of information systems). He is the Associate Editor of the IEEE TRANSACTIONS ON FUZZY SYSTEMS, Information Sciences, Advances in Fuzzy Systems, and the International Journal of Applied Metaheuristics Computing. He is a member of several journal editorial boards, including Fuzzy Sets and Systems, Applied Intelligence, Knowledge and Information Systems, Information Fusion, Evolutionary Intelligence, the International Journal of Hybrid Intelligent Systems, Memetic Computation, Swarm and Evolutionary Computation, etc. He has received many honors and awards, e.g., European Coordinating Committee for Artificial Intelligence (ECCAI) Fellow in 2009, the 2010 Spanish National Award on Computer Science ARITMEL from the "Spanish Engineer on Computer Science," and the International Cajastur "Mamdani" Prize for Soft Computing (Fourth Edition, 2010). 